\title{
Noncommutative Schwarzschild geometry and generalized uncertainty principle
}

\author{
T. Kanazawa ${ }^{1}$, G. Lambiase $^{2,3, a}$, G. Vilasi ${ }^{2}$, A. Yoshioka ${ }^{1}$ \\ ${ }^{1}$ Department of Mathematics, Faculty of Science Division II, Tokyo, University of Science, Kagurazaka 1-3, Shinjuku-ku, Tokyo 162-8601, Japan \\ ${ }^{2}$ Dipartimento di Fisica "E.R. Caianiello" Università di Salerno, 84084 Fisciano, SA, Italy \\ ${ }^{3}$ INFN, Gruppo Collegato di Salerno, Fisciano, Italy
}

Received: 11 November 2018 / Accepted: 19 January 2019 / Published online: 30 January 2019

(C) The Author(s) 2019

\begin{abstract}
We discuss a possible link between the deformation parameter $\Theta^{\mu v}$ arising in the framework of noncommutative geometry and the parameter $\beta$ of the generalized uncertainty principle (GUP). We compute the shift of the Hawking temperature induced by the $\Theta^{\mu v}$-deformed Schwarzschild geometry, and then we relate it to one obtained by GUP. Results suggest a granular structure of specetime at the Planck scales. The current bounds on $\beta$ allow to constraint the noncommutative parameter $\Theta^{\mu \nu}$.
\end{abstract}

\section{Introduction}

The possibility to describe spacetime in noncommutive frameworks was noted long time ago [1], and its interest renewed recently owing to the discovery of SeibergWitten map [2], which relates noncommutative to commutative gauge theories. Since then there has been a more and more interest to understand the impact of noncommutativity on fundamental issues. From a side by studying the the spacetime symmetry ${ }^{1}$ and unitary properties of these theories [311], from the other side, investigate on possible experimental evidences [12-14] (see the review [19,20] and references therein). Moreover, the interest increased also thanks to the fact that the low-energy limit of string theory with an antisymmetric B-field background provides a quantized structure of the spacetimes [2,19-21].

\footnotetext{
${ }^{1}$ Space-time properties of noncommutative field theories are essentially either space-time symmetries are manifestly violated [3-16], or the full Lorentz invariance is imposed on some parameters characterizing the noncommutative model, yielding to a quantum space-time with the same classical global symmetries $[6,7,17,18])$.

a e-mail: lambiase@sa.infn.it
}

The idea of noncommutativity of spacetime might provide deep indications about the quantum nature of spacetime at very high energy scales, where (gravitational) singularities are inevitable. In fact, the noncommutativity of space-time could be intrinsically connected with gravity $[2,6,7]$, and several studies have been proposed in literature to conciliate General Relativity with noncommutative space-time models. The general idea is to define the fields over phase space by replacing the ordinary product of fields with the GronewaldMoyal product and then map (via the Seiberg-Witten) this theory in the equivalent commutative theory with expansion of the fields in terms of the noncommutative parameter. This approach has been extensively used to study many gauge theories [22-28] (see also [29-32]), and since gravity can be considered as a gauge theory, the commutative equivalent approach appears to be a promising formulation ${ }^{2}[33,48,62-$ 72].

Here we shall confine ourselves to the case in which the noncommutative coordinate product is given by

$\left[x^{\mu}, x^{\nu}\right]=i \Theta^{\mu \nu}$.

\footnotetext{
2 More precisely, some formulations of General Relativity on noncommutative spacetimes have been studied in different frameworks: 1) By gauging the noncommutative $S O(4,1)$ de Sitter group and using the SeibergWitten map followed by a contraction to the Poincare group ISO $(3,1)$ [62]; 2) By twisting the Poincare algebra in such a way that the latter insures the invariance of the algebra (1.1) (canonical structure) defining the noncommutativity of the spacetime [67]; 3) By considering a restrictive class of coordinate transformations which preserve the canonical structure $[69,70]$ (by gauging the Lorentz algebra so $(3,1)$ within the enveloping algebra approach one infers a noncommutative general relativity restricted to the volume-preserving transformations (unimodular theory of gravity)); 4) By twisting the gauge Poincaré algebra [48]; 5) By considering geometrical approach to noncommutative gravity [33].
} 
The (antisymmetric) tensor $\Theta^{\mu \nu}$ is a $c$-number, with $\mu, v=$ $0, \ldots, n$, where $n+1$ is the dimension of the space-time, ${ }^{3}$ and accounts for the degree of quantum fuzziness of space-time. Although the canonical form (1.1) is the simplest case, it has the advantage to account for the basic features of noncommutativity. Hereafter we shall take 4-dimensional spacetimes. Limits on the noncommutative scale have been inferred in different frameworks, such as low-energy precision measurements [34,35,37], Lorentz symmetry violation [38,39], early Universe [40-42], black holes and gravitational physics $[43,44]$. In addition, several approaches of noncommutative theories of gravitation have been suggested [43-51,62], and all these models show that the $\Theta$-corrections occur only at the second order. More specifically, in [39], for example, it has been found that the scale of noncommutativity is limited to be smaller than the inverse $\mathrm{TeV}$ scale. Yet, the upper bounds derived from Lamb shift corrections and from cosmology (CMB physics) give $\Theta<10^{-8} \mathrm{GeV}^{-2}$ [35] and $\Theta<10^{-7}$ $\mathrm{GeV}^{-2}$ [37], respectively.

The aim of this paper is to relate the parameter $\Theta^{\mu \nu}$ to the deforming parameter $\beta$ of the generalized uncertainty principle (GUP) [58-61]. More precisely, we consider the (backreaction) effects of tiny modifications of the Schwarzschild geometry induced noncommutative geometry, which in turn affect the Hawking temperature, to the deformation of the Heisenberg uncertainty principle, that is the GUP, referring in particular to one widely studied in literature

$\Delta x \Delta p \geq \frac{\hbar}{2}\left[1+\beta\left(\frac{\Delta p}{M_{P l}}\right)^{2}\right]$,

where $M_{P l}=1.2 \times 10^{19} \mathrm{GeV}$ is the Planck mass $\left(M_{P l}^{2}=\right.$ $G^{-1}$ in natural units $\hbar=c=1$, where $G$ is the universal gravitational constant). The dimensionless parameter $\beta$ is not fixed by the theory (even if it must be pointed out that in some models of string theories it is generally assumed that $\beta \sim \mathcal{O}(1)$ [52-56]). Typically, studies in this context are mainly focalized to understand how gravity may affect the Heisenberg Uncertainty Principle, and it is therefore not surprising that the most relevant modifications have been derived in fundamental (unification) theories, such as string theory, loop quantum gravity, deformed special relativity, and black hole physics [52-56,73-78,80-82,94-99,107-112]. Our target is to use the GUP to compute the Hawking temperature of a given black hole, which however can be also computed by using the effective potential inferred by $\Theta$ deforming the Schwarzschild metric. This provides a relationship between the two parameters, $\beta \propto \Theta^{\mu \nu}$, that in turn allows to infer, by making use of the most stringent bound on

\footnotetext{
3 There are other different approaches in which the noncommutativity of the coordinates could take place, such as the Lie-algebraic and the coordinate-dependent ( $q$-deformed) formulations [57].
}

the parameter $\beta$, an upper bound on the deformation parameter $\Theta^{\mu \nu}$.

\section{GUP and Hawking temperature}

The relationship between Hawking temperature and GUP has been derived in [78, 83-90,94]. Let's shortly summarize here the main points. From Heisenberg uncertainty principle [79] it follows that the size $\delta x$ of the smallest detail of an object detectable with a beam of photons is of the order $\delta x \simeq \frac{\hbar}{2 E}$, where $E$ is the energy of photon (larger energies allow to explore smaller regions). Inserting into Eq. (1.2) one gets (for $\Delta p \simeq E$ )

$\delta x \simeq \frac{\hbar}{2 E}+2 \beta l_{P l}^{2} \frac{E}{\hbar}$

This equation allows to relate the mass $M$ and the temperature $T$ of a Schwarzschild black hole. In fact, the position uncertainty of an ensemble of unpolarized photons of Hawking radiation (just outside the event horizon of a Schwarzschild black hole $R_{S} \equiv 2 G M$ ) is of the order of $R_{S}$, and therefore the uncertainty on the photon position is $\delta x \simeq 2 \mu R_{S}$ (the constant $\mu$ is fixed in such a way to obtain the correct Hawking temperature, $\mu=\pi$ ). According to the equipartition principle, the temperature of unpolarized photons of the Hawking radiation is related to the average energy $E$ as $E=T$, so that Eq. (2.1) can be cast in the form

$M=\frac{\hbar}{8 \pi G T}+\beta \frac{T}{2 \pi}$.

The semiclassical limit $\beta \rightarrow 0$ reproduces the standard semiclassical Hawking temperature $T_{H}=\frac{\hbar}{8 \pi G M}$. The relation (2.2) is the black hole mass-temperature relation derived by making use of the GUP for a Schwarzschild black hole. By inverting (2.2) (typically $\beta T \ll 1$, in particular for solar mass black holes) one gets

$T=T_{H}\left(1+\frac{\beta}{4 \pi^{2}} \frac{M_{P l}^{2}}{M^{2}}+\cdots\right)$,

with $M_{P l}^{2}=\hbar / G$ (we set $c=1$ ). Results here derived rely on the assumption that the correction induced by the GUP has a thermal character, and, as a consequence, it can be cast in the form of a shift of $T_{H}$ (notice, however, that there exist in literature different approaches in which the corrections do not respect the exact thermality of the spectrum, as, for example, in the corpuscular model of a black hole [91]).

\section{Temperature from a $\Theta^{\mu v}$-Schwarzschild metric}

In this section, we shall derive the relation between the parameters $\Theta^{\mu \nu}$ and $\beta$. To this aim, we first recall the modifi- 
cations to the Schwarzschild metric induced by the noncommutative geometry. Then we compute the correction/shift to the Hawking temperature.

\section{$3.1 \Theta^{\mu \nu}$-Schwarzschild metric}

The question concerning the possible to find new solutions of the deformed Einstein field equations has been faced in many papers (see for example [29,30,43,44,71,72] and references therein). For our aim, we shall refer in particular to Chaichian-Tureanua-Zet paper [72], where the authors have been able to derive the noncommutative corrections $(\Theta-$ expansion) to the exact Schwarzschild solution. Essentially, the basic idea in this work is that to obtain the deformed Schwarzschild solution, one has to compute the deformed tetrad fields $\hat{e}_{\mu}^{a}(x, \Theta)$ by contracting the noncommutative gauge group $S O(4,1)$ to the Poincaré group $\operatorname{ISO}(3,1)$. In short, consider the gauge theory of de Sitter group $S O(4,1)$ on a commutative spacetime with spherical symmetry $d s^{2}=$ $g_{\mu \nu} d x^{\mu} d x^{\nu}=d r^{2}+r^{2} d \theta^{2}+r^{2} \sin ^{2} \theta d \phi^{2}-d t^{2}$. Here we are using the notation $\mu=1,2,3,0$, so that $x^{\mu}=$ $(r, \theta, \phi, t)$. The non-deformed gauge potentials are denoted with $\omega_{\mu}^{A B}(x)$, where hereafter $A, B=1,2,3,0,5$, and are identified with the spin connection $\omega_{\mu}^{a b}(x)=-\omega_{\mu}^{b a}$, and the tetrad fields $\omega_{\mu}^{a 5}(x)=k e_{\mu}^{a}(x)$, with $a, b=1,2,3,0$ and $k$ is the contraction parameter. The strength of the gauge potential $\omega_{\mu}^{A B}(x)$ is defined as

$$
\begin{aligned}
F_{\mu \nu}^{A B}=\partial_{\mu} & \hat{\omega}_{v}^{A B}(x, \Theta)-\partial_{\nu} \hat{\omega}_{v}^{A B}(x, \Theta) \\
+ & {\left[\hat{\omega}_{\mu}^{A C}(x, \Theta) \hat{\omega}_{v}^{D B}(x, \Theta)\right.} \\
& \left.-\hat{\omega}_{v}^{A C}(x, \Theta) \hat{\omega}_{\mu}^{D B}(x, \Theta)\right] \eta_{C D},
\end{aligned}
$$

where $\eta_{A B}=(1,1,1,-1,1)$. By defining $F_{\mu \nu}^{a 5}=k T_{\mu \nu}^{a}$ and $F_{\mu \nu}^{a b} \equiv R_{\mu \nu}^{a b}$, the Poincaré gauge theory assumes the geometric structure of Riemann-Cartan space $U$ (4), in which $T_{\mu \nu}^{a}$ and $R_{\mu \nu}^{a b}$ are interpreted as the torsion and curvature tensors of the Riemann-Cartan spacetime. The commutative Poincaré group theory, the $\operatorname{ISO}(3,1)$ groups, follows for $k=0$.

In the non-commutative case, in which the structure (1.1) determines the noncommutative structure of the spacetime, the noncommutative gauge theory is developed by defining the $*$-product of fields, i.e.

$$
\left.\phi(x) * \chi(x) \equiv e^{\frac{i}{2} \Theta^{\mu v} \partial_{x} \mu \partial_{y}^{v}} \phi(x) \chi(y)\right|_{y \rightarrow x} .
$$

The deformed gauge potentials are denoted with $\hat{\omega}_{\mu}^{A B}(x, \Theta)$, that fulfill the reality conditions. Expanding in terms of $\Theta^{\mu \nu}$ and using the Seiberg-Witten map, one gets

$$
\hat{\omega}_{\mu}^{A B}(x, \Theta)=\omega_{\mu}^{A B}(x)-i \Theta^{v \rho} \omega_{\mu \nu \nu \rho}^{A B}(x)
$$

$$
+\Theta^{\nu \rho} \Theta^{\alpha \sigma} \omega_{\mu \nu \rho \alpha \sigma}^{A B}(x)+\mathcal{O}\left(\Theta^{3}\right),
$$

where the coefficient $\omega_{\mu \nu \nu \rho}^{A B}(x)$ is given by

$$
\begin{aligned}
\omega_{\mu \nu v \rho}^{A B}(x) & =\frac{1}{4}\left[\omega _ { \nu } ^ { A C } \left(\partial_{\rho} \omega_{\mu}^{C B}\right.\right. \\
\left.+R_{\rho \mu}^{C B}\right) & \left.+\left(\partial_{\rho} \omega_{\mu}^{A C}+R_{\rho \mu}^{A C}\right) \omega_{v}^{C B}\right]
\end{aligned}
$$

and similar expressions for $\omega_{\mu \nu \rho \alpha \sigma}^{A B}(x)$ and other terms of the expansion, but much more involved. The limit $k \rightarrow 0$ corresponds to the $\operatorname{ISO}(3,1)$ gauge groups (hence a torsionless spacetime), and the spin connection are determined by tetrads. The deformed metric reads

$\hat{g}_{\mu \nu}(x, \Theta)=\frac{1}{2}\left(\hat{e}_{\mu}^{a} * \hat{e}_{v}^{b \dagger}+\hat{e}_{\mu}^{b} * \hat{e}_{\nu}^{a \dagger}\right) \eta_{a b}$,

where the " $"$ is the complex conjugation. As for the gauge fields, the tetrads can be expanded

$$
\begin{gathered}
\hat{e}_{\mu}^{a}(x, \Theta)=e_{\mu}^{a}(x)-i \Theta^{v \rho} e_{\mu \nu \rho}^{a}(x) \\
+\Theta^{v \rho} \Theta^{\alpha \sigma} e_{\nu \rho \alpha \sigma}^{a}(x)+\mathcal{O}\left(\Theta^{3}\right)
\end{gathered}
$$

with $e_{\mu \nu \rho}^{a}(x)=\frac{1}{4}\left[\omega_{\nu}^{a c} \partial_{\rho} e_{\mu}^{d}+\left(\partial_{\rho} \omega_{\mu}^{a c}+R_{\rho \mu}^{a c}\right) e_{\nu}^{d}\right] \eta_{c d}$, and similarly for the other terms of the expansion.

The noncommutative structure allows to derive $\Theta^{\mu \nu}$. corrections to a given geometry, in particular to the Schwarzschild geometry, to which we are interested in [71].

Following the deformation quantisation discussed in [117-120] for the Schwarzschild metric, one has to specify a Moyal algebra. Since $x^{\mu}=(r, \theta, \phi, t)$, the algebra of the functions in these variables is deformed by imposing the Moyal product (3.2) with

$\Theta^{\mu \nu}=\Upsilon\left(\begin{array}{cccc}0 & 1 & 0 & 0 \\ -1 & 0 & 0 & 0 \\ 0 & 0 & 0 & 0 \\ 0 & 0 & 0 & 0\end{array}\right)$

Here $\Upsilon$ is the deformation parameter (the ansatz (3.4) gives rise to the simplest model of noncommutativity spacetime). In the coordinate system in which $\Theta^{\mu \nu}$ assumes the form (3.4), the non vanishing component $\Upsilon$ has dimensions ${ }^{4} L$ or $E^{-1}$. Considering the non-deformed Schwarzschild geometry $d s^{2}=g_{\mu \nu}^{(S)} d x^{\mu} d x^{\nu}$, with $g_{\mu \nu}^{(S)}=\operatorname{diag}\left(A^{-1}(r), r^{2}, r^{2}\right.$ $\left.\sin ^{2} \theta,-A(r)\right), A(r)=1-\frac{\alpha}{r}(\alpha \equiv 2 G M$ and $M$ is the mass of the gravitational source), with associated vierbein fields $e_{\mu}^{1}=\left(A^{-1}, 0,0,0\right), e_{\mu}^{1}=(0, r, 0,0), e_{\mu}^{3}=$

\footnotetext{
$\overline{{ }^{4} \text { Notice that } \Upsilon} \equiv \Theta^{12}=\Theta^{r \theta}$. Consistently with results of Ref. [71], indeed, in spherical coordinates one has $x^{1}=r$ and $x^{2}=\theta$, therefore $[\Theta]=L$.
} 
$(0,0, r \sin \theta, 0)$, and $e_{\mu}^{0}=(0,0,0, A)$, one infers the components of the $\Theta$-Schwarzschild metric (see (3.3))

$\hat{g}_{\mu \nu}=g_{\mu \nu}^{(S)}+h_{\mu \nu}^{(N C)}$,

where $h_{\mu \nu}^{(N C)}$ represents the noncommutative corrections to the Schwarzschild geometry

$$
\begin{aligned}
h_{00}^{(N C)}= & -\frac{\alpha(8 r-11 \alpha)}{16 r^{4}} \Upsilon^{2}+\mathcal{O}\left(\Upsilon^{4}\right), \\
h_{r r}^{(N C)}= & -\frac{\alpha(4 r-3 \alpha)}{16 r^{2}(r-\alpha)^{2}} \Upsilon^{2}+\mathcal{O}\left(\Upsilon^{4}\right), \\
h_{\theta \theta}^{(N C)}= & \frac{2 r^{2}-17 \alpha(r-\alpha)}{32 r(r-\alpha)} \Upsilon^{2}+\mathcal{O}\left(\Upsilon^{4}\right), \\
h_{\phi \phi}^{(N C)}= & \frac{\left(r^{2}+\alpha r-\alpha^{2}\right) \cos ^{2} \theta-\alpha(2 r-\alpha)}{16 r(r-\alpha)} \Upsilon^{2} \\
& +\mathcal{O}\left(\Upsilon^{4}\right) .
\end{aligned}
$$

The limit $\Upsilon \rightarrow 0$ reproduces the standard Schwarzschild solution. Moreover, as pointed out in the Introduction, all corrections are of the second order in the deformation parameter $\Upsilon$. This is a general feature of noncommutative theories of gravitation (see for example [43-51,62]). Results here obtained are at the order $L^{2}$ due to the fact that calculations are performed in spherical coordinates ${ }^{5}$ [72,118, 120-123] (in Cartesian coordinates, ${ }^{6}$ hence in the standard canonical quantization, the non-commutative parameter $\Theta^{2}$ has dimensions $L^{4}$ ).

\subsection{Temperature shift from the deformed Schwarzschild metric}

We can now compute the shift of the Hawking temperature induced by $\Theta$-deformation algebra (3.4) (we follow the procedure delineated in $[90,94,95])$. Consider

$\hat{g}_{00}=-1+\frac{2 G M}{r}-h_{00}^{(N C)}(r)$

with $\left|h_{00}^{(N C)}(r)\right| \ll 2 G M / r$ for any $r \geq 2 G M$. The horizon's equation, i.e., $\hat{g}_{00}\left(r_{H}\right)=0$, is given by $r_{H}-$ $2 G M+r_{H} h_{00}^{(N C)}\left(r_{H}\right)=0$, and the solution is $r_{H}=$

\footnotetext{
5 Typically, in spherical coordinates one uses the notation $\sqrt{\Theta}$, with dimensions $L[72,118,120-123]$.

${ }^{6}$ It is worth to mention that in the case of modifications of the BTZ black hole in three-dimensional anti-de Sitter $\left(A d S_{3}\right)$ [124], the commutation relations are $[r, \phi]=i \hat{\theta}$, with $\hat{\theta} \equiv \theta^{r \phi}$. These differ from the Cartesian ones $\left[x^{i}, x^{j}\right]=i \theta^{i j}$ with a constant $\theta^{i j}$ since it corresponds to a nonconstant $\theta^{i j}=r \hat{\theta} \varepsilon^{i j}\left(\varepsilon^{i j}=-\varepsilon^{j i}\right)$. However, as argued in [124], the Moyal product can be still consistently defined in the polar coordinate with a constant $\theta^{r \phi}$ (spherically symmetry case).
}

$\alpha-\frac{\alpha h_{00}^{(N C)}(\alpha)}{1+\left[h_{00}^{(N C)}(\alpha)+a h_{00}^{(N C) \prime}(\alpha)\right]}$. The "deformed"Hawking temperature is given by

$$
\begin{aligned}
T=- & \hbar \frac{\hat{g}_{00}^{\prime}\left(r_{H}\right)}{4 \pi}=T_{H}\left\{1+\left[2 h_{00}^{(N C)}(r)+\alpha h_{00}^{(N C){ }^{\prime}}(r)\right]_{r=\alpha}\right\} \\
& +\mathcal{O}\left(h_{00}^{(N C) 2}\right) .
\end{aligned}
$$

where the expansion is understood in terms of $\Theta^{2}$-parameter and the symbol' stands for the derivative with respect to $\left.r{ }^{\prime} \equiv \frac{\partial}{\partial r}\right)$. By comparing the temperature (3.11) with the GUP-deformed Hawking temperature given by Eq. (2.3), one finally obtains

$\beta=\frac{4 \pi^{2} M^{2}}{M_{P l}^{2}}\left[2 h_{00}^{(N C)}(\alpha)+\alpha h_{00}^{(N C) \prime}(\alpha)\right]$,

that, by using (3.6), assumes the form

$\beta=\frac{4 \pi^{2} M^{2}}{M_{P l}^{2}}\left[-\frac{7 \Upsilon^{2}}{8 \alpha^{2}}\right]=-\frac{7 \pi^{2}}{2}\left(\Upsilon M_{P l}\right)^{2}$.

This is the wanted result, i.e. the interplay between the noncommutative deformation parameter $\Upsilon$ and the deformation parameter $\beta$ of GUP. The relation presents several interesting features:

- Remarkably, the relation between $\beta$ and $\Upsilon$ does not depend on gravitational mass $M$. This is particularly important because, as Eq. (3.13) shows, it is related to the universal character of the deformation parameter $\Theta$, suggesting its deep connection to Planck scale, then to quantum gravity.

- The above point is corroborated by the fact that $\beta \simeq$ $-\left(M_{P l} \Upsilon\right)^{2}<0$. A negative value of the GUP parameter typically arises in non-trivial space-time structures such as a (fundamental) discreteness of space, see for example [74,75,93]. Interestingly, a similar result has been also obtained in the framework of the crystal lattice [93], providing therefore a further hint that the physical spacetime could have a lattice or granular structure at the level of Planck scale.

- To infer bounds on the parameter $\Upsilon$, we require that the $\Upsilon$-correction is smaller or equal to the $\beta$-term, hence

$$
\Upsilon \leqslant \frac{2}{7 \pi^{2}} \frac{\sqrt{|\beta|}}{M_{P l}}<\frac{2}{7 \pi^{2}} \frac{\sqrt{\beta_{\exp }}}{M_{P l}},
$$

where $\beta_{\text {exp }}$ is the experimental upper bound on $|\beta|$. For the sake of completeness, in Tables 1 and 2 are reported experimental bounds on $\beta$ obtained in different frameworks. Using the the more stringent upper bound $\beta_{\text {exp }}=10^{21}$, obtained in the gravitational sector, it follows $\Upsilon<10^{-10} \mathrm{GeV}^{-1}$. Such a bound improves one order of magnitude, $\Upsilon<10^{-11} \mathrm{GeV}^{-1}$, for $\beta$ bounded from non-gravitational experiments. 
Table 1 Upper bounds on $\beta$ derived from gravitational experiments

\begin{tabular}{lll}
\hline$\beta<$ & Physical framework & Refs. \\
\hline $10^{21}$ & $\begin{array}{l}\text { Violation of equivalence principle (on } \\
\text { Earth) }\end{array}$ & {$[102](2014)$} \\
& Law of reciprocal action & \\
$10^{60}$ & GW 150914 & {$[101]$} \\
$10^{69}$ & Perihelion precession & {$[94](2015)$} \\
& (Solar system data) & \\
$10^{71}$ & $\begin{array}{l}\text { Perihelion precession } \\
\end{array}$ & (Pulsar PRS B 1913+16 data) \\
$10^{78}$ & Modified mass-temperature relation & {$[94](2015)$} \\
& Light deflection & \\
\hline
\end{tabular}

Table 2 Upper bounds on $\beta$ derived from non-gravitational experiments

\begin{tabular}{|c|c|c|}
\hline$\beta<$ & Physical framework & Refs. \\
\hline $10^{18}$ & $\begin{array}{l}\text { Evolution of micro and nano } \\
\text { mechanical oscillators (masses } \\
\sim m_{\mathrm{p}} \text { ) }\end{array}$ & [103] (2015) \\
\hline $10^{20}$ & Lamb shift & [104-106] (2011) \\
\hline $10^{21}$ & Scanning tunneling microscope & {$[104-106](2008)$} \\
\hline $10^{33}$ & Gravitational bar detectors $^{\mathrm{a}}$ & [100] (2013) \\
\hline $10^{34}$ & Electroweak measurement & [104-106] (2011) \\
\hline $10^{34}$ & $\begin{array}{l}\text { Charmonium levels } \\
\text { Energy difference in Hydrogen } \\
\text { levels } 1 S-2 S\end{array}$ & $\begin{array}{l}{[104-106](2011)} \\
{[113](2010)}\end{array}$ \\
\hline $10^{39}$ & ${ }^{87} \mathrm{Rb}$ cold-atom-recoil experiment & [114] (2016) \\
\hline $10^{46}$ & Landau levels & [104-106] (2011) \\
\hline
\end{tabular}

This bound is derived without explicitly involving the gravitational interaction

- As pointed out in the Introduction, the deformation parameter $\beta$ is not fixed by the theory, and it is generally assumed that $\beta \sim \mathcal{O}(1-10)$, as suggested by some models of string theory [52-56]. It is hence interesting to observe that if the parameter $\Upsilon$ is of the order of the Planck scale, $\Upsilon \sim M_{P l}^{-1}$ (the quantum gravity scale), then the GUP deformation parameter $\beta$ can be fixed to the value $|\beta| \sim \frac{7 \pi^{2}}{2} \sim \mathcal{O}(1-10)$.

\section{Conclusions}

In this paper we have derived an upper bound on the deformation parameter $\Upsilon$ of the noncommutative geometry (referring in particular to the gravitational sector of noncommutative geometry), by relating $\Upsilon$ to the coefficients $\beta$ of GUP. The shift of the Hawking temperature, for which the GUP is relevant, is derived by means of pure quantum mechan- ics principles, and no specific representations of canonical commutator relation is postulated. On the other hand, the same temperature is derived geometrically for a deformed Schwarzschild metric, allowing to link the deformed uncertainty relation with the $\Theta$-deformed metric. We have found that the $\Theta^{2}$-correction to the canonical commutation relations of Heisenberg algebra is negative, suggesting a discrete nature of spacetime at the Planck scales, and that the more stringent bound that the current experiments allow to obtain is $\Upsilon<10^{-11}-10^{-10}$ (here $\Upsilon \equiv \Theta^{12}=\Theta^{r \theta}$ ).

Here we focused on noncommutative geometry putting attention to the gravitational sector, but understanding whenever other algebras may affect GUP, or specific representations of canonical operators, is certainly a non trivial task, especially for the possible links with quantum gravity. There is indeed a wide discussion on the implications of various models yielding GUPs, and a common aspect of all these models is related to test the size of these modifications. These aspects appear particularly interesting in perspective of laboratory-scale imitation of the black hole horizon, with the subsequent possible emission of an analogue Hawking radiation $[115,116]$.

Acknowledgements GL and GV thank Prof. A. Yoshioka and Dr. T. Kanazawa for the kind hospitality, and the Tokyo University of Science for support. GL thanks F. Scardigli for discussions and suggestions. The authors acknowledge the referee for very constructive comments.

Data Availability Statement This manuscript has no associated data or the data will not be deposited. [Authors' comment: Our study only involves theoretical analysis. The manuscript has no associated data.]

Open Access This article is distributed under the terms of the Creative Commons Attribution 4.0 International License (http://creativecomm ons.org/licenses/by/4.0/), which permits unrestricted use, distribution, and reproduction in any medium, provided you give appropriate credit to the original author(s) and the source, provide a link to the Creative Commons license, and indicate if changes were made. Funded by SCOAP . $^{3}$

\section{References}

1. H. Snyder, Phys. Rev. 71, 38 (1947)

2. N. Seiberg, E. Witten, J. High Energy Phys. 09, 32 (1999)

3. L. Alvarez-Gaumé, M.A. Vazquez-Mozo, Nucl. Phys. B 668, 293 (2003)

4. S. Carroll, J. Harvey, V.A. Kostelecky, C. Lane, T. Okamoto, Phys. Rev. Lett. 87, 141601 (2001)

5. M. Chaichian, K. Nishijima, A. Tureanu, Phys. Lett. B 568, 146 (2003)

6. S. Doplicher, in Proceedings of the 37th Karpacz Winter School of Theoretical Physics, 2001, p. 204, hep-th/0105251;

7. S. Doplicher, K. Fredenhagen, J.E. Roberts, Commun. Math. Phys. 172, 187 (1995)

8. D. Bahns, S. Doplicher, K. Fredenhagen, G. Piacitelli, Phys. Lett. B 533, 178 (2002)

9. A. Iorio, T. Sykora, Int. J. Mod. Phys. A 17, 2369 (2002)

10. R. Jackiw, S.Y. Pi, Phys. Rev. Lett. 88, 111603 (2002) 
11. R. Jackiw, ibid. 41, 1635 (1978)

12. G. Amelino-Camelia, G. Mandanici, K. Yoshida, On the IR/UV mixing and experimental limits on the parameters of canonical noncommutative spacetimes, arxiv:hep-th/0209254

13. Z. Guralnik, R. Jackiw, S.Y. Pi, A.P. Polychronakos, Phys. Lett. B 517, 450 (2001)

14. R.G. Cai, ibid. 517, 457 (2001)

15. J.M. Grimstrup, B. Kloibock, L. Popp, V. Putz, M. Schweda, M. Wickenhauser, The energy-momentum tensor in noncommutative gauge field models, arxiv:hep-th/0210288

16. A.A. Bichl, J.M. Grimstrup, H. Grosse, E. Kraus, L. Popp, M. Schweda, R. Wulkenhaar, Eur. Phys. J. C 24, 165 (2002)

17. M.R. Douglas, N.A. Nekrasov, Rev. Mod. Phys. 73, 977 (2001)

18. H. Omori, Y. Maeda, N. Miyazaki, A. Yoshioka, Lett. Math. Phys. 82, 153 (2007)

19. M.R. Douglas, N.A. Nekrasov, Rev. Mod. Phys. 73, 977 (2001)

20. R.J. Szabo, Phys. Rep. 378, 207 (2003)

21. F. Ardalan, H. Arfaei, M.M. Sheikh-Jabbari, J. High Energy Phys. 02, 016 (1999)

22. O.F. Dayi, B. Yapiskann, J. High Energy Phys. 10, 022 (2002)

23. S. Ghosh, Nucl. Phys. B 670, 359 (2003)

24. B. Chakraborty, S. Gangopadhyay, A. Saha, Phys. Rev. D 70, 107707 (2004)

25. S. Ghosh, Phys. Rev. D 70, 085007 (2004)

26. P. Mukherjee, A. Saha, Mod. Phys. Lett. A 21, 821 (2006)

27. A. Saha, A. Rahaman, P. Mukherjee, Phys. Lett. B 638, 292 (2006)

28. X. Calmet, A. Kobakhidze, Phys. Rev. D 72, 045010 (2005)

29. M. Chaichian, P. Presnajder, M.M. Sheikh-Jabbari, A. Tureanu, Eur. Phys. J. C 29, 413 (2003)

30. M. Chaichian, A. Kobakhidze, A. Tureanu, Eur. Phys. J. C 47, 241 (2006)

31. X. Calmet, B. Jurco, P. Schupp, J. Wess, M. Wohlgenannt, Eur. Phys. J. C 23, 363 (2002)

32. P. Aschieri, B. Jurco, P. Schupp, J. Wess, Nucl. Phys. B 651, 45 (2003)

33. M. Chaichian, A. Tureanu, R.B. Zhang, X. Zhang, J. Math. Phys. 49, 073511 (2008)

34. I. Mocioiu, M. Pospelov, R. Roiban, Phys. Lett. B 489, 390 (2000)

35. M. Chaichian, M. Sheikh-Jabbari, A. Tureanu, Phys. Rev. Lett. 86, 2716 (2001)

36. M. Chaichian, M. Sheikh-Jabbari, A. Tureanu, Eur. Phys. J. C 36, 251 (2004)

37. P.K. Joby, P. Chingangbam, S. Das, Phys. Rev. D 91, 083503 (2015)

38. S.M. Carroll, J.A. Harvey, V.A. Kostelecky, C.D. Lane, T. Okamoto, Phys. Rev. Lett. 87, 141601 (2001)

39. X. Calmet, Eur. Phys. J. C 41, 269 (2005)

40. P. Joby, P. Chingangbam, S. Das, Phys. Rev. D 91, 083503 (2015)

41. X. Calmet, C. Fritz, Phys. Lett. B 747, 406 (2015)

42. G. Lambiase, G. Vilasi, A. Yoshioka, Class. Quantum Gravity 34, 025004 (2017)

43. A. Kobakhidze, C. Lagger, A. Manning, Phys. Rev. D 94, 064033 (2016)

44. A. Kobakhidze, Phys. Rev. D 79, 047701 (2009)

45. P. Aschieri, C. Blohmann, M. Dimitrijevic, F. Meyer, P. Schupp, J. Wess, Class. Quantum Gravity 22, 3511 (2005)

46. X. Calmet, A. Kobakhidze, Phys. Rev. D 72, 045010 (2005)

47. P. Aschieri, M. Dimitrijevic, F. Meyer, J. Wess, Class. Quantum Gravity 23, 1883 (2006)

48. A. Kobakhidze, Int. J. Mod. Phys. A 23, 2541 (2008)

49. R.J. Szabo, Class. Quantum Gravity 23, R199 (2006)

50. X. Calmet, A. Kobakhidze, Phys. Rev. D 74, 047702 (2006)

51. P. Mukherjee, A. Saha, Phys. Rev. D 74, 027702 (2006)

52. D. Amati, M. Ciafaloni, G. Veneziano, Phys. Lett. B 197, 81 (1987)

53. D.J. Gross, P.F. Mende, Phys. Lett. B 197, 129 (1987)
54. D. Amati, M. Ciafaloni, G. Veneziano, Phys. Lett. B 216, 41 (1989)

55. K. Konishi, G. Paffuti, P. Provero, Phys. Lett. B 234, 276 (1990)

56. S. Capozziello, G. Lambiase, G. Scarpetta, Int. J. Theor. Phys. 39, $15(2000)$

57. J. Madore, S. Schraml, P. Schupp, J. Wess, Eur. Phys. J. C 16, 161 (2000)

58. H.S. Snyder, Phys. Rev. 71, 38 (1947)

59. C.N. Yang, Phys. Rev. 72, 874 (1947)

60. C.A. Mead, Phys. Rev. 135, B849 (1964)

61. F. Karolyhazy, Nuovo Cim. A 42, 390 (1966)

62. A.H. Chamseddine, Phys. Lett. B 504, 33 (2001)

63. L. Bonora, M. Schnabl, M. Sheikh-Jabbari, A. Tomasiello, Nucl. Phys. B 589, 461 (2000)

64. B. Jurco, S. Schraml, P. Schupp, J. Wess, Eur. Phys. J. C 17, 521 (2000)

65. M. Chaichian, P.P. Kulish, K. Nishijima, A. Tureanu, Phys. Lett. B 604, 98 (2004)

66. M. Chaichian, P. Prenajder, A. Tureanu, Phys. Rev. Lett. 94, $151602(2005)$

67. P. Aschieri, C. Blohmann, M. Dimitrijevic, F. Meyer, P. Schupp, J. Wess, Class. Quantum Gravity 22, 3511 (2005)

68. L. lvarez-Gaum, F. Meyer, M.A. Vazquez-Mozo, Nucl. Phys. B 75, 392 (2006)

69. X. Calmet, A. Kobakhidze, Phys. Rev. D 72, 045010 (2005)

70. X. Calmet, A. Kobakhidze, Phys. Rev. D 74, 047702 (2006)

71. M. Chaichian, A. Tureanu, Phys. Lett. B 637, 199 (2006)

72. M. Chaichian, A. Tureanu, G. Zet, Phys. Lett. B 660, 573 (2008)

73. M. Maggiore, Phys. Lett. B 304, 65 (1993)

74. A. Kempf, G. Mangano, R.B. Mann, Phys. Rev. D 52, 1108 (1995)

75. M. Bojowald, A. Kempf, Phys. Rev. D 86, 085017 (2012)

76. F. Scardigli, Phys. Lett. B 452, 39 (1999)

77. R.J. Adler, D.I. Santiago, Mod. Phys. Lett. A 14, 1371 (1999)

78. F. Scardigli, R. Casadio, Class. Quantum Gravity 20, 3915 (2003)

79. W. Heisenberg, Zeitschrift für Physik 43, 172 (1927)

80. K. Nozari, B. Fazlpour, Chaos Solitons Fractals 34, 224 (2007)

81. K. Nozari, Phys. Lett. B 629, 41 (2005)

82. K. Nozari, T. Azizi, Gen. Relativ. Gravit. 38, 735 (2006)

83. F. Scardigli, Nuovo Cim. B 110, 1029 (1995)

84. R.J. Adler, P. Chen, D.I. Santiago, Gen. Relativ. Gravit. 33, 2101 (2001)

85. M. Cavaglia, S. Das, Class. Quantum Gravity 21, 4511 (2004)

86. M. Cavaglia, S. Das, R. Maartens, Class. Quantum Gravity 20, L205 (2003)

87. L. Susskind, J. Lindesay, An Introduction to Black Holes, Information, and the String Theory Revolution (World Scientific, Singapore, 2005). See chapter 10

88. K. Nouicer, Class. Quantum Gravity 24, 5917 (2007)

89. F. Scardigli, Glimpses on the micro black hole planck phase. arXiv:0809.1832

90. F. Scardigli, G. Lambiase, E. Vagenas, Phys. Lett. B 767, 242 (2017)

91. G. Dvali, C. Gomez, Black Hole's Quantum N-Portrait. arXiv:1112.3359

92. S. Weinberg, Gravitation and Cosmology (Wiley, New York, 1972)

93. P. Jizba, H. Kleinert, F. Scardigli, Phys. Rev. D 81, 084030 (2010)

94. F. Scardigli, R. Casadio, Eur. Phys. J. C 75, 425 (2015)

95. G. Lambiase, F. Scardigli, Phys. Rev. D 97, 075003 (2018)

96. F. Scardigli, G. Lambiase, E. Vagenas, Phys. Lett. B 767, 242 (2017)

97. F. Scardigli, G. Lambiase, E. Vagenas, J. Phys. Conf. Ser. 880, 012044 (2017)

98. F. Scardigli, M. Blasone, G. Luciano, R. Casadio, Eur. Phys. J. C 78, 728 (2018)

99. A.N. Tawfik, A.M. Diab, Rep. Prog. Phys. 78, N. 12 (2015) 
100. F. Marin, M. Cerdonio et al., Nat. Phys. 9, 71 (2013)

101. Z.-W. Feng, S.-Z. Yang, H.-L. Li, X.-T. Zu, Phys. Lett. B 768, 81 (2017)

102. S. Ghosh, Class. Quantum Gravity 31, 025025 (2014)

103. M. Bawaj, C. Biancofiore, F. Marin, Nat. Commun. 6, 7503 (2015). arXiv:1411.6410

104. S. Das, E.C. Vagenas, Phys. Rev. Lett. 101, 221301 (2008)

105. A.F. Ali, S. Das, E.C. Vagenas, Phys. Rev. D 84, 044013 (2011)

106. S. Das, E.C. Vagenas Can. J. Phys. 84, 233 (2009)

107. E.C. Vagenas, L. Alasfar, S.M. Alsaleh, A.F. Ali, Nucl. Phys. B 931, $72(2018)$

108. E.C. Vagenas, S.M. Alsaleh, A. Farag, EPL 120, 40001 (2017)

109. M. Faizal, A.F. Ali, A. Nassar, Phys. Lett. B 765, 238 (2017)

110. L. Perivolaropoulos, Phys. Rev. D 95, 103523 (2017)

111. A. Alonso-Serrano, M.P. Dabrowski, H. Gohar, Phys. Rev. D 97, 044029 (2018)

112. B. Khosropour, M. Eghbali, S. Ghorbanali, Gen. Relativ. Gravit. 50, 25 (2018)

113. C. Quesne, V.M. Tkachuk, Phys. Rev. A 81, 012106 (2010)

114. D. Gao, M. Zhan, Phys. Rev. A 94, 013607 (2016)
115. J. Steinhauer, Nat. Phys. 10, 864 (2014)

116. R. Cowen, Nature News, 12 October 2014, Hawking radiation mimicked in the lab, https://doi.org/10.1038/nature.2014.16131

117. M. Chaichian, A. Tureanu, R.B. Zhang, X. Zhang, J. Math. Phys. 49, 073511 (2008)

118. D. Wang, R.B. Zhang, X. Zhang, Class. Quantum Gravity 26, 085014 (2009)

119. D. Wang, R.B. Zhang, X. Zhang, Eur. Phys. J. C 64, 439 (2009)

120. W. Sun, D. Wang, N. Xie, R.B. Zhang, X. Zhang, Eur. Phys. J. C 69, 271 (2010)

121. M. Chaichian, A. Tureanu, M.R. Setare, G. Zet, JHEP04, 064 (2008)

122. D.V. Singh, M.S. Ali, S.G. Ghosh, Int. J. Mod. Phys. D 27, 1850108 (2018)

123. S.A. Alavi, S. Nodeh, Phys. Scr. 90, 035301 (2015)

124. H-C. Kim, Mu-In Park, C. Rim, J.H. Yee, JHEP 0810060 (2008) 